\title{
Inequalities for Estimations of Integrals Related to Higher-Order Strongly $n$-Polynomial Preinvex Functions
}

\author{
Yongping Deng (D), ${ }^{1}$ Muhammad Uzair Awan (D), ${ }^{2}$ Sadia Talib, ${ }^{2}$ Muhammad Aslam Noor (D), \\ Khalida Inayat Noor, ${ }^{3}$ Pshtiwan Othman Mohammed $\mathbb{D}^{4},{ }^{4}$ and Shanhe Wu $\mathbb{D}^{1}$ \\ ${ }^{1}$ Department of Mathematics, Longyan University, Longyan 364012, China \\ ${ }^{2}$ Department of Mathematics, Government College University, Faisalabad, Pakistan \\ ${ }^{3}$ Department of Mathematics, COMSATS University Islamabad, Islamabad, Pakistan \\ ${ }^{4}$ Department of Mathematics, University of Sulaimani, Sulaimani, Kurdistan Region, Iraq
}

Correspondence should be addressed to Shanhe Wu; shanhewu@gmail.com

Received 27 September 2020; Revised 29 October 2020; Accepted 5 November 2020; Published 22 December 2020

Academic Editor: Xiaolong Qin

Copyright (c) 2020 Yongping Deng et al. This is an open access article distributed under the Creative Commons Attribution License, which permits unrestricted use, distribution, and reproduction in any medium, provided the original work is properly cited.

\begin{abstract}
In this paper, we establish an integral identity associated with $m$-times differentiable functions. The result is then used to derive some integral estimations for higher-order strongly $n$-polynomial preinvex functions. Finally, we apply the obtained inequalities to construct new inequalities involving special means.
\end{abstract}

\section{Introduction}

The convexity properties of functions have been a powerful tool for dealing with various problems of pure and applied sciences. In recent decades, the concept of classical convexity has been extended and generalized in different directions, and the study relevant to this subject has stimulated an increased interest. In [1], Weir and Mond introduced the notion of preinvexity as follows.

Definition 1. A set $\mathscr{X} \subset \mathbb{R}$ is said to be invex with respect to $\xi: \mathbb{R} \times \mathbb{R} \longrightarrow \mathbb{R}$ if

$$
e+\tau \xi(f, e) \in \mathscr{X}, \quad \forall e, f \in \mathscr{X}, \tau \in[0,1]
$$

Definition 2. A function $\Lambda: \mathscr{X} \longrightarrow \mathbb{R}$ is said to be preinvex with respect to $\xi: \mathbb{R} \times \mathbb{R} \longrightarrow \mathbb{R}$ if

$$
\begin{gathered}
\Lambda(e+\tau \xi(f, e)) \leq(1-\tau) \Lambda(e)+\tau \Lambda(f), \\
\forall e, f \in \mathscr{X}, \tau \in[0,1] .
\end{gathered}
$$

For $\xi(f, e)=f-e$, the class of preinvex functions reduces to the class of convex functions. It had been shown that the preinvex functions may not be convex functions. For example, the function $\Lambda=-|x|$ is not a convex function, but it is a preinvex function with respect to $\xi$ provided that

$$
\xi(f, e)= \begin{cases}f-e, & \text { if } f \leq 0, e \leq 0 \text { and } f \geq 0, e \geq 0, \\ e-f, & \text { otherwise. }\end{cases}
$$

Mohan and Neogy [2] and Mititelu [3] discussed the properties of invex sets and preinvex functions. Noor [4] and Noor et al. [5] investigated integral inequalities of Hermite-Hadamard type for log-preinvex functions and $h$-preinvex functions, respectively. Wu et al. [6] considered harmonically $(p, h, m)$-preinvex functions and relevant inequalities. Peng et al. [7] established fractional Simpson's inequalities with the aid of $\left(m, h_{1}, h_{2}\right)$-preinvexity. Du et al. [8] dealt with integral inequalities involving generalized $m$-convex functions on fractal sets. Saleh [9] defined $(\varepsilon, m)$-preinvex function and studied the properties of $(\varepsilon, m)$-preinvex functions. Mohammed [10] focused on the integral inequalities for preinvex functions via the generalized beta function. 
Very recently, Toplu et al. [11] introduced and investigated the class of $n$-polynomial convex functions as follows.

Definition 3. Let $n \in \mathbb{N}$. A nonnegative function $\Lambda: I \subset \mathbb{R} \longrightarrow \mathbb{R}$ is called an $n$-polynomial convex function if for any $e, f \in I$ and $\tau \in[0,1]$,

$$
\begin{aligned}
\Lambda(\tau e+(1-\tau) f) \leq & \frac{1}{n} \sum_{s=1}^{n}\left[1-(1-\tau)^{s}\right] \Lambda(e) \\
& +\frac{1}{n} \sum_{s=1}^{n}\left[1-\tau^{s}\right] \Lambda(f) .
\end{aligned}
$$

Note that if we take $n=1$ in the above inequality, then we have 1-polynomial convexity which is just the classical convexity. It is also necessary to mention here that every nonnegative convex function is also an $n$-polynomial convex function. For more properties on the $n$-polynomial convex function, see [11].

Kadakal [12] and Karamardian [13] put forward the class of strongly convex functions, respectively. Strong convexity is just the strengthening version of convexity, which reads as follows.

Definition 4. A function $\Lambda: I \subset \mathbb{R} \longrightarrow \mathbb{R}$ is said to be a strongly convex function with modulus $\mu>0$ if

$$
\begin{aligned}
& \Lambda((1-\tau) e+\tau f) \leq(1-\tau) \Lambda(e) \\
&+\tau \Lambda(f)-\mu \tau(1-\tau)|f-e|^{2}, \\
& \forall e, f \in I, \tau \in[0,1] .
\end{aligned}
$$

In order to deal with the problems of mathematical programs with equilibrium constraints, Lin and Fukushima [14] proposed a generalization of the strongly convex function, which is called the higher-order strongly convex functions.

Definition 5. A function $\Lambda: I \subset \mathbb{R} \longrightarrow \mathbb{R}$ is said to be a strongly convex function with order $\sigma>0$ and modulus $\mu>0$ if

$$
\begin{aligned}
& \Lambda((1-\tau) e+\tau f) \leq(1-\tau) \Lambda(e) \\
&+\tau \Lambda(f)-\mu \tau(1-\tau)|f-e|^{\sigma}, \\
& \forall e, f \in I, \tau \in[0,1] .
\end{aligned}
$$

The higher-order strongly convex functions would reduce to the strongly convex function in the special case when $\sigma=2$.

As an extension of classical convex functions, the strongly convex functions have been widely used in establishing new inequalities and dealing with estimations of integrals. Angulo et al. [15] and Zhang et al. [16] presented some inequalities for strongly $h$-convex functions and strongly $(s, m)$-convex functions, respectively. In order to further generalize the strongly convex functions, recently, some researchers, such as Mishra and Sharma [17], Noor and Noor [18], and Mohsen et al. [19], began to study the higher-order strongly convex functions. Wu et al. [20, 21] gave some estimates of the upper bound for differentiable functions associated with $k$-fractional integrals and higherorder strongly convex functions. Khan et al. [22] and Ullah et al. [23] introduced the concept of coordinate and the technique of majorization into the study of strongly convex functions.

In this paper, we introduce the notion for a new class of strongly convex functions which are named as the strongly $n$-polynomial preinvex functions of order $\sigma>0$.

Definition 6. Let $n \in \mathbb{N}$. A nonnegative function $\Lambda: \mathscr{X} \longrightarrow \mathbb{R}$ is said to be a strongly $n$-polynomial preinvex function of order $\sigma>0$ with modulus $\mu>0$ if

$$
\begin{aligned}
\Lambda(e+(1-\tau) \xi(f, e)) \leq & \frac{1}{n} \sum_{s=1}^{n}\left[1-(1-\tau)^{s}\right] \Lambda(e) \\
& +\frac{1}{n} \sum_{s=1}^{n}\left[1-\tau^{s}\right] \Lambda(f) \\
& -\mu\left[\tau^{\sigma}(1-\tau)+\tau(1-\tau)^{\sigma}\right]|\xi(f, e)|^{\sigma}
\end{aligned}
$$

holds for all $e, f \in \mathscr{X}$ and $\tau \in[0,1]$.

Remark 1. Note that

(i) If we take $\sigma=2$ in Definition 6, then we have the class of strongly $n$-polynomial preinvex functions

(ii) If we take $\xi(f, e)=f-e$ in Definition 6 , then we have the class of strongly $n$-polynomial convex functions of order $\sigma>0$

(iii) If we take $\xi(f, e)=f-e$ and $\sigma=2$ in Definition 6, then we have the class of strongly $n$-polynomial convex functions

(iv) If we take $n=1$ in Definition 6 , then we have the class of strongly preinvex functions of order $\sigma>0$

This shows that the class of strongly $n$-polynomial preinvex functions of order $\sigma>0$ is quite unifying one as it relates several unrelated classes of convexity.

The main objective of this paper is to establish a new integral identity for $m$-times differentiable functions. With the help of this identity, we deduce some inequalities for estimations of integrals associated with $m$-times differentiable strongly preinvex functions of order $\sigma>0$. As applications, we discuss some special cases of the main results and establish several new inequalities involving special means.

\section{A Key Lemma}

Here, we establish an auxiliary result which will play a significant role in the development of our main results.

Lemma 1. Let $\Lambda:[e, e+\xi(f, e)] \longrightarrow \mathbb{R}$ be an m-times differentiable function on $(e, e+\xi(f, e))$ with $\xi(f, e)>0$. If $\Lambda^{(m)} \in L[e, e+\xi(f, e)]$, then the following integral equality holds for $m \in \mathbb{N}_{+}$: 


$$
\begin{aligned}
& \frac{1}{\xi(f, e)} \int_{e}^{e+\xi(f, e)} \Lambda(x) \mathrm{d} x-\sum_{k=1}^{m} \frac{\xi^{k-1}(f, e)\left[\Lambda^{(k-1)}(e)+(-1)^{k-1} \Lambda^{(k-1)}(e+\xi(f, e))\right]}{2 k !} \\
& =\frac{\xi^{m}(f, e)}{2 m !} \int_{0}^{1}\left[\tau^{m}+(\tau-1)^{m}\right] \Lambda^{(m)}(e+(1-\tau) \xi(f, e)) \mathrm{d} \tau .
\end{aligned}
$$

Proof. Integrating by parts, we have

$$
\begin{aligned}
& \frac{\xi^{m+1}(f, e)}{2 m !} \int_{0}^{1} \tau^{m} \Lambda^{(m)}(e+(1-\tau) \xi(f, e)) \mathrm{d} \tau \\
& =-\frac{\xi^{m}(f, e)}{2 m !} \Lambda^{(m-1)}(e)+\frac{\xi^{m}(f, e)}{2(m-1) !} \int_{0}^{1} \tau^{m-1} \Lambda^{(m-1)}(e+(1-\tau) \xi(f, e)) \mathrm{d} \tau \\
& =-\frac{\xi^{m}(f, e)}{2 m !} \Lambda^{(m-1)}(e)-\frac{\xi^{m-1}(f, e)}{2(m-1) !} \Lambda^{(m-2)}(e)+\frac{\xi^{m-1}(f, e)}{2(m-2) !} \int_{0}^{1} \tau^{m-2} \Lambda^{(m-2)}(e+(1-\tau) \xi(f, e)) \mathrm{d} \tau \\
& =-\sum_{k=1}^{m-1} \frac{\xi^{k+1}(f, e) \Lambda^{(k)}(e)}{2(k+1) !}+\frac{\xi^{2}(f, e)}{2} \int_{0}^{1} \tau \Lambda^{\prime}(e+(1-\tau) \xi(f, e)) \mathrm{d} \tau \\
& =-\sum_{k=1}^{m} \frac{\xi^{k}(f, e) \Lambda}{2 k !}(k-1)(e) \\
& \hline
\end{aligned}
$$

Similarly, we deduce that

$$
\begin{aligned}
& \frac{\xi^{m+1}(f, e)}{2 m !} \int_{0}^{1}(\tau-1)^{m} \Lambda^{(m)}(e+(1-\tau) \xi(f, e)) \mathrm{d} \tau \\
& =\frac{\xi^{m}(f, e)}{2 m !}(-1)^{m} \Lambda^{(m-1)}(e+\xi(f, e))+\frac{\xi^{m}(f, e)}{2(m-1) !} \int_{0}^{1}(\tau-1)^{m-1} \Lambda^{(m-1)}(e+(1-\tau) \xi(f, e)) \mathrm{d} \tau \\
& =\frac{\xi^{m}(f, e)}{2 m !}(-1)^{m} \Lambda^{(m-1)}(e+\xi(f, e))+\frac{\xi^{m-1}(f, e)}{2(m-1) !}(-1)^{m-1} \Lambda^{(m-2)}(e+\xi(f, e)) \\
& \quad+\frac{\xi^{m-1}(f, e)}{2(m-2) !} \int_{0}^{1}(\tau-1)^{m-2} \Lambda^{(m-2)}(e+(1-\tau) \xi(f, e)) \mathrm{d} \tau \\
& =\sum_{k=1}^{m-1} \frac{\xi^{k+1}(f, e)(-1)^{k+1} \Lambda^{(k)}(e+\xi(f, e))}{2(k+1) !}+\frac{\xi^{2}(f, e)}{2} \int_{0}^{1}(\tau-1) \Lambda^{\prime}(e+(1-\tau) \xi(f, e)) \mathrm{d} \tau \\
& =\sum_{k=1}^{m} \frac{\xi^{k}(f, e)(-1)^{k} \Lambda^{(k-1)}(e+\xi(f, e))}{2 k !}+\frac{1}{2} \int_{e}^{e+\xi(f, e)} \Lambda(x) \mathrm{d} x .
\end{aligned}
$$


Combining (9) and (10) completes the proof of Lemma 1.

\section{Main Results}

We are now in a position to state our main results.
Theorem 1. Let $\Lambda:[e, e+\xi(f, e)] \longrightarrow \mathbb{R}$ be an m-times differentiable function on $(e, e+\xi(f, e))$ with $\xi(f, e)>0$ and $\Lambda^{(m)} \in L[e, e+\xi(f, e)]$. If $\left|\Lambda^{(m)}\right|$ is a strongly $n$-polynomial preinvex function of order $\sigma>0$ with modulus $\mu>0$, then

$$
\begin{aligned}
& \left|\frac{1}{\xi(f, e)} \int_{e}^{e+\xi(f, e)} \Lambda(x) \mathrm{d} x-\sum_{k=1}^{m} \frac{\xi^{k-1}(f, e)\left[\Lambda^{(k-1)}(e)+(-1)^{k-1} \Lambda^{(k-1)}(e+\xi(f, e))\right]}{2 k !}\right| \\
& \quad \leq \frac{\xi^{m}(f, e)}{2 m !}\left[\frac{\left|\Lambda^{(m)}(e)\right|}{n} \sum_{s=1}^{n} \mathscr{H}_{1}+\frac{\left|\Lambda^{(m)}(f)\right|}{n} \sum_{s=1}^{n} \mathscr{H}_{1}-\mu \mathscr{H}_{2} \xi^{\sigma}(f, e)\right],
\end{aligned}
$$

where

$$
\begin{aligned}
& \mathscr{H}_{1}=\frac{m+2 s+1}{(m+1)(m+s+1)}-B(m+1, s+1), \\
& \mathscr{H}_{2}=\frac{2}{(m+\sigma+1)(m+\sigma+2)}+2 B(\sigma+1, m+2),
\end{aligned}
$$

and $B(x, y)=\int_{0}^{1} \tau^{x-1}(1-\tau)^{y-1} d \tau$ is the beta function.

Proof. Using Lemma 1 and the fact that $\left|\Lambda^{(m)}\right|$ is a strongly $n$-polynomial preinvex function of order $\sigma>0$, we have

$$
\begin{aligned}
& \left|\frac{1}{\xi(f, e)} \int_{e}^{e+\xi(f, e)} \Lambda(x) \mathrm{d} x-\sum_{k=1}^{m} \frac{\xi^{k-1}(f, e)\left[\Lambda^{(k-1)}(e)+(-1)^{k-1} \Lambda^{(k-1)}(e+\xi(f, e))\right]}{2 k !}\right| \\
& \leq \frac{\xi^{m}(f, e)}{2 m !} \int_{0}^{1}\left[\tau^{m}+(1-\tau)^{m}\right]\left|\Lambda^{(m)}(e+(1-\tau) \xi(f, e))\right| \mathrm{d} \tau \\
& \leq \frac{\xi^{m}(f, e)}{2 m !} \int_{0}^{1}\left[\tau^{m}+(1-\tau)^{m}\right]\left[\frac{1}{n} \sum_{s=1}^{n}\left[1-(1-\tau)^{s}\right]\left|\Lambda^{(m)}(e)\right|+\frac{1}{n} \sum_{s=1}^{n}\left[1-\tau^{s}\right]\left|\Lambda^{(m)}(f)\right|-\mu \xi^{\sigma}(f, e)\left[\tau^{\sigma}(1-\tau)+\tau(1-\tau)^{\sigma}\right]\right] \mathrm{d} \tau \\
& =\frac{\xi^{m}(f, e)}{2 m !}\left[\frac{\left|\Lambda^{(m)}(e)\right|}{n} \int_{0}^{1}\left[\tau^{m}+(1-\tau)^{m}\right] \sum_{s=1}^{n}\left[1-(1-\tau)^{s}\right] \mathrm{d} \tau+\frac{\left|\Lambda^{(m)}(f)\right|}{n}\right. \\
& \left.\cdot \int_{0}^{1}\left[\tau^{m}+(1-\tau)^{m}\right] \sum_{s=1}^{n}\left[1-\tau^{s}\right] \mathrm{d} \tau-\mu \xi^{\sigma}(f, e) \int_{0}^{1}\left[\tau^{m}+(1-\tau)^{m}\right]\left[\tau^{\sigma}(1-\tau)+\tau(1-\tau)^{\sigma}\right] \mathrm{d} \tau\right] \\
& =\frac{\xi^{m}(f, e)}{2 m !}\left[\frac{\left|\Lambda^{(m)}(e)\right|}{n} \sum_{s=1}^{n} \int_{0}^{1}\left[\tau^{m}+(1-\tau)^{m}\right]\left[1-(1-\tau)^{s}\right] \mathrm{d} \tau+\frac{\left|\Lambda^{(m)}(f)\right|}{n} \sum_{s=1}^{n}\right. \\
& \left.\cdot \int_{0}^{1}\left[\tau^{m}+(1-\tau)^{m}\right]\left[1-\tau^{s}\right] \mathrm{d} \tau-\mu \xi^{\sigma}(f, e) \int_{0}^{1}\left[\tau^{m}+(1-\tau)^{m}\right]\left[\tau^{\sigma}(1-\tau)+\tau(1-\tau)^{\sigma}\right] \mathrm{d} \tau\right]
\end{aligned}
$$


where

$$
\begin{aligned}
\int_{0}^{1}\left[\tau^{m}+(1-\tau)^{m}\right]\left[1-(1-\tau)^{s}\right] \mathrm{d} \tau & =\frac{m+2 s+1}{(m+1)(m+s+1)}-B(m+1, s+1)=\mathscr{H}_{1}, \\
\int_{0}^{1}\left[\tau^{m}+(1-\tau)^{m}\right]\left[1-\tau^{s}\right] \mathrm{d} \tau & =\frac{m+2 s+1}{(m+1)(m+s+1)}-B(s+1, m+1)=\mathscr{H}_{1}, \\
\int_{0}^{1}\left[\tau^{m}+(1-\tau)^{m}\right]\left[\tau^{\sigma}(1-\tau)+\tau(1-\tau)\right]^{\sigma} \mathrm{d} \tau & =\frac{2}{(m+\sigma+1)(m+\sigma+2)}+2 B(\sigma+1, m+2)=\mathscr{H}_{2} .
\end{aligned}
$$

The proof of Theorem 1 is completed.

In the following, we discuss some special cases of Theorem 1.

(1) If we take the limit $\mu \longrightarrow 0$ in Theorem 1 , we get a result for the $n$-polynomial preinvex function.
Corollary 1. Under the assumptions of Theorem 1, if $\left|\Lambda^{(m)}\right|$ is an n-polynomial preinvex function, then

$$
\begin{aligned}
& \left|\frac{1}{\xi(f, e)} \int_{e}^{e+\xi(f, e)} \Lambda(x) \mathrm{d} x-\sum_{k=1}^{m} \frac{\xi^{k-1}(f, e)\left[\Lambda^{(k-1)}(e)+(-1)^{k-1} \Lambda^{(k-1)}(e+\xi(f, e))\right]}{2 k !}\right| \\
& \quad \leq \frac{\xi^{m}(f, e)}{2 m !}\left[\frac{\left|\Lambda^{(m)}(e)\right|}{n} \sum_{s=1}^{n} \mathscr{H}_{1}+\frac{\left|\Lambda^{(m)}(f)\right|}{n} \sum_{s=1}^{n} \mathscr{H}_{1}\right],
\end{aligned}
$$

where

$$
\mathscr{H}_{1}=\frac{m+2 s+1}{(m+1)(m+s+1)}-B(m+1, s+1) .
$$

Corollary 2. Under the assumptions of Theorem 1, if $\left|\Lambda^{(m)}\right|$ is a strongly n-polynomial preinvex function, then

(2) If we take $\sigma=2$ in Theorem 1, we get a result for the strongly $n$-polynomial preinvex function.

$$
\begin{aligned}
& \left|\frac{1}{\xi(f, e)} \int_{e}^{e+\xi(f, e)} \Lambda(x) \mathrm{d} x-\sum_{k=1}^{m} \frac{\xi^{k-1}(f, e)\left[\Lambda^{(k-1)}(e)+(-1)^{k-1} \Lambda^{(k-1)}(e+\xi(f, e))\right]}{2 k !}\right| \\
& \quad \leq \frac{\xi^{m}(f, e)}{2 m !}\left[\frac{\left|\Lambda^{(m)}(e)\right|}{n} \sum_{s=1}^{n} \mathscr{H}_{1}+\frac{\left|\Lambda^{(m)}(f)\right|}{n} \sum_{s=1}^{n} \mathscr{H}_{1}-\mu \mathscr{H}_{3} \xi^{2}(f, e)\right]
\end{aligned}
$$

where

$$
\begin{aligned}
& \mathscr{H}_{1}=\frac{m+2 s+1}{(m+1)(m+s+1)}-B(m+1, s+1) \\
& \mathscr{H}_{3}=\int_{0}^{1}\left[\tau^{m}+(1-\tau)^{m}\right]\left[\tau^{2}(1-\tau)+\tau(1-\tau)^{2}\right] \mathrm{d} \tau=\frac{2}{(m+2)(m+4)} .
\end{aligned}
$$

(3) If we take $n=1$ in Theorem 1 , we get a result for the higher-order strongly preinvex function.
Corollary 3. Under the assumptions of Theorem 1, if $\left|\Lambda^{(m)}\right|$ is a strongly preinvex function of order $\sigma>0$, then 


$$
\begin{aligned}
& \left|\frac{1}{\xi(f, e)} \int_{e}^{e+\xi(f, e)} \Lambda(x)-\sum_{k=1}^{m} \frac{\xi^{k-1}(f, e)\left[\Lambda^{(k-1)}(e)+(-1)^{k-1} \Lambda^{(k-1)}(e+\xi(f, e))\right]}{2 k !}\right| \\
& \quad \leq \frac{\xi^{m}(f, e)}{2 m !}\left[\frac{\left|\Lambda^{(m)}(e)\right|+\left|\Lambda^{(m)}(f)\right|}{m+1}-\mu \mathscr{H}_{2} \xi^{\sigma}(f, e)\right] .
\end{aligned}
$$

Theorem 2. Let $\Lambda:[e, e+\xi(f, e)] \longrightarrow \mathbb{R}$ be an m-times differentiable function on $(e, e+\xi(f, e))$ with $\xi(f, e)>0,1 /$ $p+1 / q=1, \quad p>1, q>1$, and $\Lambda^{(m)} \in L[e, e+\xi(f, e)]$. If
$\left|\Lambda^{(m)}\right|^{q}$ is a strongly n-polynomial preinvex function of order $\sigma>0$ with modulus $\mu>0$, then

$$
\begin{aligned}
& \left|\frac{1}{\xi(f, e)} \int_{e}^{e+\xi(f, e)} \Lambda(x) \mathrm{d} x-\sum_{k=1}^{m} \frac{\xi^{k-1}(f, e)\left[\Lambda^{(k-1)}(e)+(-1)^{k-1} \Lambda^{(k-1)}(e+\xi(f, e))\right]}{2 k !}\right| \\
& \quad \leq \frac{\xi^{m}(f, e)}{2 m !}\left(\frac{2^{p}}{m p+1}\right) \frac{1}{p}\left(\frac{2}{n} \sum_{s=1}^{n} \frac{s}{s+1} \mathscr{A}\left(\left|\Lambda^{(m)}(e)\right|^{q},\left|\Lambda^{(m)}(f)\right|^{q}\right)-\frac{2 \mu \xi^{\sigma}(f, e)}{(\sigma+1)(\sigma+2)}\right)^{1 / q},
\end{aligned}
$$

where $\mathscr{A}(.,$.$) is the arithmetic mean.$

$$
\begin{aligned}
& \left|\frac{1}{\xi(f, e)} \int_{e}^{e+\xi(f, e)} \Lambda(x) \mathrm{d} x-\sum_{k=1}^{m} \frac{\xi^{k-1}(f, e)\left[\Lambda^{(k-1)}(e)+(-1)^{k-1} \Lambda^{(k-1)}(e+\xi(f, e))\right]}{2 k !}\right| \\
& \quad \leq \frac{\xi^{m}(f, e)}{2 m !} \int_{0}^{1}\left[\tau^{m}+(1-\tau)^{m}\right]\left|\Lambda^{(m)}(e+(1-\tau) \xi(f, e))\right| \mathrm{d} \tau \\
& \quad \leq \frac{\xi^{m}(f, e)}{2 m !}\left(\int_{0}^{1}\left[\tau^{m}+(1-\tau)^{m}\right]^{p} \mathrm{~d} \tau\right)^{1 / p}\left(\int_{0}^{1}\left|\Lambda^{(m)}(e+(1-\tau) \xi(f, e))\right|^{q} \mathrm{~d} \tau\right)^{1 / q} \\
& \quad \leq \frac{\xi^{m}(f, e)}{2 m !}\left(\int_{0}^{1} 2^{p-1}\left[\tau^{m p}+(1-\tau)^{m p}\right] \mathrm{d} \tau\right)^{1 / p}\left(\int_{0}^{1}\left|\Lambda^{(m)}(e+(1-\tau) \xi(f, e))\right|^{q} \mathrm{~d} \tau\right)^{1 / q} \\
& \quad \leq \frac{\xi^{m}(f, e)}{2 m !}\left(\frac{2^{p}}{m p+1}\right)^{1 / p}\left(\int_{0}^{1} \frac{1}{n} \sum_{s=1}^{n}\left[1-(1-\tau)^{s}\right]\left|\Lambda^{(m)}(e)\right|^{q} \mathrm{~d} \tau\right. \\
& \left.\quad+\int_{0}^{1} \frac{1}{n} \sum_{s=1}^{n}\left[1-\tau^{s}\right]\left|\Lambda^{(m)}(f)\right|^{q} \mathrm{~d} \tau-\mu \xi^{\sigma}(f, e) \int_{0}^{1}\left[\tau^{\sigma}(1-\tau)+\tau(1-\tau)^{\sigma}\right] \mathrm{d} \tau\right)^{1 / q}
\end{aligned}
$$

Proof. Utilizing Lemma 1, Hölder's integral inequality, and the fact that $\left|\Lambda^{(m)}\right|^{q}$ is a strongly $n$-polynomial preinvex function of order $\sigma>0$, one has 


$$
\begin{aligned}
& =\frac{\xi^{m}(f, e)}{2 m !}\left(\frac{2^{p}}{m p+1}\right)^{1 / p}\left(\frac{\left|\Lambda^{(m)}(e)\right|^{q}}{n} \int_{0}^{1} \sum_{s=1}^{n}\left[1-(1-\tau)^{s}\right] \mathrm{d} \tau\right. \\
& \left.+\frac{\left|\Lambda^{(m)}(f)\right|^{q}}{n} \int_{0}^{1} \sum_{s=1}^{n}\left[1-\tau^{s}\right] \mathrm{d} \tau-\mu \xi^{\sigma}(f, e) \int_{0}^{1}\left[\tau^{\sigma}(1-\tau)+\tau(1-\tau)^{\sigma}\right] \mathrm{d} \tau\right)^{1 / q} \\
& =\frac{\xi^{m}(f, e)}{2 m !}\left(\frac{2^{p}}{m p+1}\right)^{1 / p}\left(\frac{\left|\Lambda^{(m)}(e)\right|^{q}}{n} \sum_{s=1}^{n} \int_{0}^{1}\left[1-(1-\tau)^{s}\right] \mathrm{d} \tau\right. \\
& \left.+\frac{\left|\Lambda^{(m)}(f)\right|^{q}}{n} \sum_{s=1}^{n} \int_{0}^{1}\left[1-\tau^{s}\right] \mathrm{d} \tau-\mu \xi^{\sigma}(f, e) \int_{0}^{1}\left[\tau^{\sigma}(1-\tau)+\tau(1-\tau)^{\sigma}\right] \mathrm{d} \tau\right) \\
& =\frac{\xi^{m}(f, e)}{2 m !}\left(\frac{2^{p}}{m p+1}\right)^{1 / p}\left(\frac{2}{n} \sum_{s=1}^{n} \frac{s}{s+1} \mathscr{A}\left(\left|\Lambda^{(m)}(e)\right|^{q},\left|\Lambda^{(m)}(f)\right|^{q}\right)-\frac{2 \mu \xi^{\sigma}(f, e)}{(\sigma+1)(\sigma+2)}\right)^{1 / q} .
\end{aligned}
$$

This completes the proof of Theorem 2 .

In the following, we discuss some special cases of Theorem 2.

(1) Putting $\mu \longrightarrow 0$ in Theorem 2, we obtain a result for the $n$-polynomial preinvex function.

$$
\begin{array}{r}
\left|\frac{1}{\xi(f, e)} \int_{e}^{e+\xi(f, e)} \Lambda(x) \mathrm{d} x-\sum_{k=1}^{m} \frac{\xi^{k-1}(f, e)\left[\Lambda^{(k-1)}(e)+(-1)^{k-1} \Lambda^{(k-1)}(e+\xi(f, e))\right]}{2 k !}\right| \\
\leq \frac{\xi^{m}(f, e)}{2 m !}\left(\frac{2^{p}}{m p+1}\right)^{1 / p}\left(\frac{2}{n} \sum_{s=1}^{n} \frac{s}{s+1} \mathscr{A}\left(\left|\Lambda^{(m)}(e)\right|^{q},\left|\Lambda^{(m)}(f)\right|^{q}\right)\right)^{1 / q} .
\end{array}
$$

(2) Putting $\sigma=2$ in Theorem 2, we obtain a result for the strongly $n$-polynomial preinvex function.
Corollary 5. Under the assumptions of Theorem 2 , if $\left|\Lambda^{(m)}\right|^{q}$ is a strongly n-polynomial preinvex function, then

$$
\begin{aligned}
& \left|\frac{1}{\xi(f, e)} \int_{e}^{e+\xi(f, e)} \Lambda(x) \mathrm{d} x-\sum_{k=1}^{m} \frac{\xi^{k-1}(f, e)\left[\Lambda^{(k-1)}(e)+(-1)^{k-1} \Lambda^{(k-1)}(e+\xi(f, e))\right]}{2 k !}\right| \\
& \leq \frac{\xi^{m}(f, e)}{2 m !}\left(\frac{2^{p}}{m p+1}\right)^{1 / p}\left(\frac{2}{n} \sum_{s=1}^{n} \frac{s}{s+1} \mathcal{A}\left(\left|\Lambda^{(m)}(e)\right|^{q},\left|\Lambda^{(m)}(f)\right|^{q}\right)-\frac{\mu|\xi(f, e)|^{2}}{6}\right)^{1 / q} .
\end{aligned}
$$

(3) Putting $n=1$ in Theorem 2, we obtain a result for the higher-order strongly preinvex function.
Corollary 6. Under the assumptions of Theorem 2 , if $\left|\Lambda^{(m)}\right|^{q}$ is a strongly preinvex function of order $\sigma>0$, then 


$$
\begin{aligned}
& \left|\frac{1}{\overline{\xi(f, e)}} \int_{e}^{e+\xi(f, e)} \Lambda(x) \mathrm{d} x-\sum_{k=1}^{m} \frac{\xi^{k-1}(f, e)\left[\Lambda^{(k-1)}(e)+(-1)^{k-1} \Lambda^{(k-1)}(e+\xi(f, e))\right]}{2 k !}\right| \\
& \quad \leq \frac{\xi^{m}(f, e)}{2 m !}\left(\frac{2^{p}}{m p+1}\right)^{1 / p}\left(\mathscr{A}\left(\left|\Lambda^{(m)}(e)\right|^{q},\left|\Lambda^{(m)}(f)\right|^{q}\right)-\frac{2 \mu \xi^{\sigma}(f, e)}{(\sigma+1)(\sigma+2)}\right)^{1 / q} .
\end{aligned}
$$

Theorem 3. Let $\Lambda:[e, e+\xi(f, e)] \longrightarrow \mathbb{R}$ be an m-times differentiable function on $(e, e+\xi(f, e))$ with $\xi(f, e)>0, q \geq 1$, and $\Lambda^{(m)} \in L[e, e+\xi(f, e)]$. If $\left|\Lambda^{(m)}\right|^{q}$ is a strongly n-polynomial preinvex function of order $\sigma>0$ with modulus $\mu>0$, then

$$
\begin{aligned}
& \left|\frac{1}{\xi(f, e)} \int_{e}^{e+\xi(f, e)} \Lambda(x) \mathrm{d} x-\sum_{k=1}^{m} \frac{\xi^{k-1}(f, e)\left[\Lambda^{(k-1)}(e)+(-1)^{k-1} \Lambda^{(k-1)}(e+\xi(f, e))\right]}{2 k !}\right| \\
& \quad \leq \frac{\xi^{m}(f, e)}{2 m !}\left(\frac{2}{m+1}\right)^{1-1 / q}\left(\frac{\left|\Lambda^{(m)}(e)\right|^{q}}{n} \sum_{s=1}^{n} \mathscr{H}_{1}+\frac{\left|\Lambda^{(m)}(f)\right|^{q}}{n} \sum_{s=1}^{n} \mathscr{H}_{1}-\mu \mathscr{H}_{2} \xi^{\sigma}(f, e)\right)^{1 / q},
\end{aligned}
$$

where $\mathscr{H}_{1}$ and $\mathscr{H}_{2}$ are the expressions as described in Theorem 1.
Proof. Applying Lemma 1, power-mean integral inequality, and the fact that $\left|\Lambda^{(m)}\right|^{q}$ is a strongly $n$-polynomial preinvex function of order $\sigma>0$, we have

$$
\begin{aligned}
& \left|\frac{1}{\bar{\xi}(f, e)} \int_{e}^{e+\xi(f, e)} \Lambda(x) \mathrm{d} x-\sum_{k=1}^{m} \frac{\xi^{k-1}(f, e)\left[\Lambda^{(k-1)}(e)+(-1)^{k-1} \Lambda^{(k-1)}(e+\xi(f, e))\right]}{2 k !}\right| \\
& \quad \leq \frac{\xi^{m}(f, e)}{2 m !} \int_{0}^{1}\left[\tau^{m}+(1-\tau)^{m}\right]\left|\Lambda^{(m)}(e+(1-\tau) \xi(f, e))\right| \mathrm{d} \tau \\
& \quad \leq \frac{\xi^{m}(f, e)}{2 m !}\left(\int_{0}^{1}\left[\tau^{m}+(1-\tau)^{m}\right] \mathrm{d} \tau\right)^{1-1 / q} \times\left(\int_{0}^{1}\left[\tau^{m}+(1-\tau)^{m}\right]\left|\Lambda^{(m)}(e+(1-\tau) \xi(f, e))\right|^{q} \mathrm{~d} \tau\right)^{1 / q} \\
& \quad \leq \frac{\xi^{m}(f, e)}{2 m !}\left(\int_{0}^{1}\left[\tau^{m}+(1-\tau)^{m}\right] \mathrm{d} \tau\right)^{1-1 / q} \\
& \quad \times\left(\int_{0}^{1}\left[\tau^{m}+(1-\tau)^{m}\right]\left[\frac{1}{n} \sum_{s=1}^{n}\left[1-(1-\tau)^{s}\right]\left|\Lambda^{(m)}(e)\right|^{q}+\frac{1}{n} \sum_{s=1}^{n}\left\{\begin{array}{c}
{\left[1-\tau^{s}\right]\left|\Lambda^{(m)}(f)\right|^{q}-\mu \xi^{\sigma}(f, e)} \\
\times\left[\tau^{\sigma}(1-\tau)+\tau(1-\tau)^{\sigma}\right]
\end{array}\right\}\right] \mathrm{d} \tau\right)^{1 / q}
\end{aligned}
$$




$$
\begin{aligned}
& =\frac{\xi^{m}(f, e)}{2 m !}\left(\frac{2}{m+1}\right)^{1-1 / q}\left(\begin{array}{c}
\frac{\left|\Lambda^{(m)}(e)\right|^{q}}{n} \int_{0}^{1}\left[\tau^{m}+(1-\tau)^{m}\right] \sum_{s=1}^{n}\left[1-(1-\tau)^{s}\right] \mathrm{d} \tau+\frac{\left|\Lambda^{(m)}(f)\right|^{q}}{n} \int_{0}^{1}\left[\tau^{m}+(1-\tau)^{m}\right] \\
\sum_{s=1}^{n}\left[1-\tau^{s}\right] \mathrm{d} \tau-\mu \xi^{\sigma}(f, e) \int_{0}^{1}\left[\tau^{m}+(1-\tau)^{m}\right]\left[\tau^{\sigma}(1-\tau)+\tau(1-\tau)^{\sigma}\right] \mathrm{d} \tau
\end{array}\right) \\
& =\frac{\xi^{m}(f, e)}{2 m !}\left(\frac{2}{m+1}\right)^{1-1 / q}\left(\begin{array}{c}
\frac{\left|\Lambda^{(m)}(e)\right|^{q}}{n} \sum_{s=1}^{n} \int_{0}^{1}\left[\tau^{m}+(1-\tau)^{m}\right]\left[1-(1-\tau)^{s}\right] \mathrm{d} \tau+\frac{\left|\Lambda^{(m)}(f)\right|^{q}}{n} \\
\sum_{s=1}^{n} \int_{0}^{1}\left[\tau^{m}+(1-\tau)^{m}\right]\left[1-\tau^{s}\right] \mathrm{d} \tau \\
-\mu \xi^{\sigma}(f, e) \int_{0}^{1}\left[\tau^{m}+(1-\tau)^{m}\right]\left[\tau^{\sigma}(1-\tau)+\tau(1-\tau)^{\sigma}\right] \mathrm{d} \tau
\end{array}\right) \\
& =\frac{\xi^{m}(f, e)}{2 m !}\left(\frac{2}{m+1}\right)^{1-1 / q}\left(\frac{\left|\Lambda^{(m)}(e)\right|^{q}}{n} \sum_{s=1}^{n} \mathscr{H}_{1}+\frac{\left|\Lambda^{(m)}(f)\right|^{q}}{n} \sum_{s=1}^{n} \mathscr{H}_{1}-\mu \mathscr{H}_{2} \xi^{\sigma}(f, e)\right)^{1 / q} .
\end{aligned}
$$

Theorem 3 is proved.

Finally, we discuss some special cases of Theorem 3.

(1) Letting $\mu \longrightarrow 0$ in Theorem 3, we get a result for the $n$-polynomial preinvex function.
Corollary 7. Under the assumptions of Theorem 3, if $\left|\Lambda^{(m)}\right|^{q}$ is an n-polynomial preinvex function of order $\sigma>0$, then

$$
\begin{array}{r}
\left|\frac{1}{\xi(f, e)} \int_{e}^{e+\xi(f, e)} \Lambda(x) \mathrm{d} x-\sum_{k=1}^{m} \frac{\xi^{k-1}(f, e)\left[\Lambda^{(k-1)}(e)+(-1)^{k-1} \Lambda^{(k-1)}(e+\xi(f, e))\right]}{2 k !}\right| \\
\quad \leq \frac{\xi^{m}(f, e)}{2 m !}\left(\frac{2}{m+1}\right)^{1-1 / q}\left(\frac{\left|\Lambda^{(m)}(e)\right|^{q}}{n} \sum_{s=1}^{n} \mathscr{H}_{1}+\frac{\left|\Lambda^{(m)}(f)\right|^{q}}{n} \sum_{s=1}^{n} \mathscr{H}_{1}\right)^{1 / q} .
\end{array}
$$

(2) Choosing $\sigma=2$ in Theorem 3, we get a result for the strongly $n$-polynomial preinvex function.
Corollary 8. Under the assumptions of Theorem 3, if $\left|\Lambda^{(m)}\right|^{q}$ is a strongly n-polynomial preinvex function, then

$$
\begin{aligned}
& \left|\frac{1}{\xi(f, e)} \int_{e}^{e+\xi(f, e)} \Lambda(x) \mathrm{d} x-\sum_{k=1}^{m} \frac{\xi^{k-1}(f, e)\left[\Lambda^{(k-1)}(e)+(-1)^{k-1} \Lambda^{(k-1)}(e+\xi(f, e))\right]}{2 k !}\right| \\
& \leq \frac{\xi^{m}(f, e)}{2 m !}\left(\frac{2}{m+1}\right)^{1-1 / q}\left(\frac{\left|\Lambda^{(m)}(e)\right|^{q}}{n} \sum_{s=1}^{n} \mathscr{H}_{1}+\frac{\left|\Lambda^{(m)}(f)\right|^{q}}{n} \sum_{s=1}^{n} \mathscr{H}_{1}-\mu \mathscr{H}_{3}|\xi(f, e)|^{2}\right)^{1 / q} .
\end{aligned}
$$


(3) Choosing $n=1$ in Theorem 3, we get a result for the higher-order strongly preinvex function.
Corollary 9. Under the assumptions of Theorem 3, if $\left|\Lambda^{(m)}\right|^{q}$ is a strongly preinvex function of order $\sigma>0$, then

$$
\begin{array}{r}
\left|\frac{1}{\xi(f, e)} \int_{e}^{e+\xi(f, e)} \Lambda(x) \mathrm{d} x-\sum_{k=1}^{m} \frac{\xi^{k-1}(f, e)\left[\Lambda^{(k-1)}(e)+(-1)^{k-1} \Lambda^{(k-1)}(e+\xi(f, e))\right]}{2 k !}\right| \\
\quad \leq \frac{\xi^{m}(f, e)}{2 m !}\left(\frac{2}{m+1}\right)^{1-1 / q}\left(\frac{\left|\Lambda^{(m)}(e)\right|^{q}+\left|\Lambda^{(m)}(f)\right|^{q}}{m+1}-\mu \mathscr{H}_{2} \xi^{\sigma}(f, e)\right)^{1 / q} .
\end{array}
$$

\section{Application to Special Means}

We consider the following special means.

(1) The arithmetic mean:

$$
\mathscr{A}(e, f)=\frac{e+f}{2}
$$

(2) The mean of the function $\Psi$ on $[e, f]$

$$
\mathscr{A}_{\Psi}(e, f)=\frac{1}{f-e} \int_{e}^{f} \Psi(e) \mathrm{d} x .
$$

Proposition 1. Let $\Psi:[e, e+\xi(f, e)] \rightarrow \mathbb{R}$ be m-times differentiable function on $(e, e+\xi(f, e))$ with $\xi(f, e)>0$ and $\Psi^{(m)} \in L[e, e+\xi(f, e)]$. If $\left|\Psi^{(m)}\right|$ is a strongly $n$-polynomial preinvex function of order $\sigma>0$ with modulus $\mu>0$, then the following inequality holds:

$$
\begin{aligned}
& \left|\mathscr{A}_{\Psi}(e, e+\xi(f, e))-\sum_{k=1}^{m} \frac{\xi^{k-1}(f, e)}{k !} \mathscr{A}\left(\Psi^{(k-1)}(e),(-1)^{k-1} \Psi^{(k-1)}(e+\xi(f, e))\right)\right| \\
& \quad \leq \frac{\xi^{m}(f, e)}{2 m !}\left(\frac{2}{n} \sum_{s=1}^{n} \mathscr{H}_{1} \mathscr{A}\left(\Psi^{(m)}(e), \Psi^{(m)}(f)\right)-\mu \mathscr{H}_{2} \xi^{\sigma}(f, e)\right),
\end{aligned}
$$

where $\mathscr{H}_{1}$ and $\mathscr{H}_{2}$ are as in Theorem 1.

Proof. Taking $\Lambda(x)=\Psi(x), x \in[e, e+\xi(f, e)]$ and using Theorem 1, we get the desired result (32).
Proposition 2. Let $\Psi:[e, e+\xi(f, e)] \rightarrow \mathbb{R}$ be m-times differentiable function on $(e, e+\xi(f, e))$ with $\xi(f, e)>0$, $1 / p+1 / q=1, p>1, q>1$ and $\Psi^{(m)} \in L[e, e+\xi(f, e)]$. If $\left|\Psi^{(m)}\right|^{q}$ is a strongly n-polynomial preinvex function of order $\sigma>0$ with modulus $\mu>0$, then the following inequality holds:

$$
\begin{aligned}
& \left|\mathscr{A}_{\Psi}(e, e+\xi(f, e))-\sum_{k=1}^{m} \frac{\xi^{k-1}(f, e)}{k !} \mathscr{A}\left(\Psi^{(k-1)}(e),(-1)^{k-1} \Psi^{(k-1)}(e+\xi(f, e))\right)\right| \\
& \quad \leq \frac{\xi^{m}(f, e)}{2 m !}\left(\frac{2^{p}}{m p+1}\right)^{1 / p}\left(\frac{2}{n} \sum_{s=1}^{n} \frac{s}{s+1} \mathscr{A}\left(\left|\Psi^{(m)}(e)\right|^{q},\left|\Psi^{(m)}(f)\right|^{q}\right)-\frac{2 \mu \xi^{\sigma}(f, e)}{(\sigma+1)(\sigma+2)}\right)^{1 / q} .
\end{aligned}
$$


Proof. Setting $\Lambda(x)=\Psi(x), x \in[e, e+\xi(f, e)]$ and applying Theorem 2, we get the desired result (33).

Proposition 3. Let $\Psi:[e, e+\xi(f, e)] \rightarrow \mathbb{R}$ be m-times differentiable function on $(e, e+\xi(f, e))$ with $\xi(f, e)>0, q \geq 1$ and $\Psi^{(m)} \in L[e, e+\xi(f, e)]$. If $\left|\Psi^{(m)}\right|^{q}$ is a strongly n-polynomial preinvex function of order $\sigma>0$ with modulus $\mu>0$, then the following inequality holds:

$$
\begin{aligned}
& \left|\mathscr{A}_{\Psi}(e, e+\xi(f, e))-\sum_{k=1}^{m} \frac{\xi^{k-1}(f, e)}{k !} \mathscr{A}\left(\Psi^{(k-1)}(e),(-1)^{k-1} \Psi^{(k-1)}(e+\xi(f, e))\right)\right| \\
& \leq \frac{\xi^{m}(f, e)}{2 m !}\left(\frac{2}{m+1}\right)^{1-1 / q}\left(\frac{2}{n} \sum_{s=1}^{n} \mathscr{H}_{1} \mathscr{A}\left(\left|\Psi^{(m)}(e)\right|^{q},\left|\Psi^{(m)}(f)\right|^{q}\right)-\mu \mathscr{H}_{2} \xi^{\sigma}(f, e)\right)^{1 / q},
\end{aligned}
$$

where $\mathscr{H}_{1}$ and $\mathscr{H}_{2}$ are as in Theorem 1.

Proof. Taking $\Lambda(x)=\Psi(x), x \in[e, e+\xi(f, e)]$ and utilizing Theorem 3 , we get the desired result (34).

\section{Data Availability}

No data were used to support this study.

\section{Conflicts of Interest}

The authors declare that there are no conflicts of interest regarding the publication of this paper.

\section{Authors' Contributions}

All authors contributed equally and significantly to this paper. All authors read and approved the final manuscript.

\section{Acknowledgments}

This work was supported by the Natural Science Foundation of Fujian Province of China (Grant no. 2020J01365) and the Teaching Reform Project of Fujian Provincial Education Department (Grant no. FBJG20180120).

\section{References}

[1] T. Weir and B. Mond, "Pre-invex functions in multiple objective optimization," Journal of Mathematical Analysis and Applications, vol. 136, no. 1, pp. 29-38, 1988.

[2] S. R. Mohan and S. K. Neogy, "On invex sets and preinvex functions," Journal of Mathematical Analysis and Applications, vol. 189, no. 3, pp. 901-908, 1995.

[3] S. Mititelu, "Invex sets," Student Cercamics Materials, vol. 46, no. 5, pp. 529-532, 1994.

[4] M. A. Noor, "Hermite-Hadamard integral inequalities for logpreinvex functions," Journal of Mathematical Analysis and Approximation Theory, vol. 2, pp. 126-131, 2007.

[5] M. Noor, K. Noor, M. Awan, and J. Li, "On hermite-hadamard inequalities for $h$-preinvex functions," Filomat, vol. 28, no. 7, pp. 1463-1474, 2014.

[6] S. Wu, I. A. Baloch, and I. Ișcan, "On harmonically $(p, h, m)$-preinvex functions," Journal of Function Spaces, vol. 2017, Article ID 2148529, , 2017.
[7] C. Peng, C. Zhou, and T. S. Du, "Riemann-liouville fractional Simpson's inequalities through generalized $\left(m, h_{1}, h_{2}\right)$ - preinvexity," Italian Journal of Pure and Applied Mathematics, vol. 38, pp. 345-367, 2017.

[8] T. S. Du, H. Wang, M. A. Khan, and Y. Zhang, "Certain integral inequalities considering generalized $m$-convexity on fractal sets and their applications," Fractals, vol. 27, no. 7, pp. 1-17, Article ID 1950117, 2019.

[9] W. Saleh, "Some properties of $(\varepsilon, m)$-preinvex functions," Communications in Optimization Theory, vol. 2020, p. 8, 2020.

[10] P. O. Mohammed, "New integral inequalities for preinvex functions via generalized beta function," Journal of Interdisciplinary Mathematics, vol. 22, no. 4, pp. 539-549, 2019.

[11] T. Toplu, M. Kadakal, I.' Işcan, and İ. İşcan, "On $n$-polynomial convexity and some related inequalities," AIMS Mathematics, vol. 5, no. 2, pp. 1304-1318, 2020.

[12] B. T. Kadakal, "Existence theorems and convergence of minimizing sequences in extremum problems with restrictions," Soviet Mathematics-Doklady, vol. 7, pp. 72-75, 1966.

[13] S. Karamardian, "The nonlinear complementarity problem with applications, part 2," Journal of Optimization Theory and Applications, vol. 4, no. 3, pp. 167-181, 1969.

[14] G. H. Lin and M. Fukushima, "Some exact penalty results for nonlinear programs and mathematical programs with equilibrium constraints," Journal of Optimization Theory and Applications, vol. 118, no. 1, pp. 67-80, 2003.

[15] H. Angulo, J. Gimenez, A. M. Moros, and K. Nikodem, "On strongly $h$-convex functions," Annals of Functional Analysis, vol. 2, no. 2, pp. 85-91, 2011.

[16] J. Zhang, Z. L. Pei, and F. Qi, "Integral inequalities of Simpson's type for strongly extended $(s, m)$-convex functions," Journal of Computational Analysis and Applications, vol. 26, no. 3, pp. 499-508, 2019.

[17] S. K. Mishra and N. Sharma, "On strongly generalized convex functions of higher order," Mathematical Inequalities \& Applications, vol. 22, no. 1, pp. 111-121, 2019.

[18] M. A. Noor and K. I. Noor, "Higher order strongly general convex functions and variational inequalities," AIMS Mathematics, vol. 5, no. 4, pp. 3646-3663, 2020.

[19] B. B. Mohsen, M. A. Noor, and K. I. Noor, "Strongly convex functions of higher order involving bifunction," Mathematics, vol. 7, no. 11, p. 1028, 2019.

[20] S. Wu, M. U. Awan, and M. V. Mihai, "Estimates of upper bound for a $k$-th order differentiable functions involving Riemann-Liouville integrals via higher order strongly h-preinvex functions," Journal of Inequalities and Applications, vol. 2019, p. 227, 2019. 
[21] S. Wu, M. U. Awan, and Z. Javed, "Estimates of upper bound for differentiable functions associated with $k$-fractional integrals and higher order strongly s-convex functions," Journal of Function Spaces, vol. 2020, Article ID 5091857, , 2020.

[22] M. A. Khan, S. Z. Ullah, and Y. M. Chu, "The concept of coordinate strongly convex functions and related inequalities," Revista de la Real Academia de Ciencias Exactas, Fisicas y Naturales-Series A: Matematicas, vol. 113, no. 3, pp. 22352251, 2019.

[23] S. Z. Ullah, M. D. Khan, and Y. M. Chu, "Majorization theorems for strongly convex functions," Journal of Inequalities and Applications, vol. 2019, p. 58, 2019. 